\title{
What's the message? Interpretation of an uninformative BRCA1/2 test result for women at risk of familial breast cancer
}

Sandra van Dijk, $M A^{1}$, Wilma Otten, $M A, P h D^{1}$, Daniëlle R.M. Timmermans, $M A, P h D^{3}$, Christi J. van Asperen, $M D, P h D^{2}$, Hanne Meijers-Heijboer, $M D, P h D^{4}$, Aad Tibben, $M A, P h D^{2}$, Martijn H. Breuning, $M D, P h D^{2}$, and Job Kievit, $M D, P h D^{1}$

\begin{abstract}
Purpose: To test the "false-reassurance hypothesis," which suggests that women who receive an uninformative $B R C A 1 / 2$ test result may incorrectly conclude that they no longer have an elevated risk, with possible harmful consequences for adherence to breast surveillance guidelines. Methods: A prospective questionnaire design was used to compare 183 women with an uninformative BRCA test result (94 affected and 89 unaffected) with 41 proven $B R C A$ mutation-carriers and 49 true negatives before and after BRCA1/2 test disclosure. Results: After DNA-test disclosure, test applicants differed from each other with regard to their perception of the likelihood of carrying a deleterious gene $(P<0.0001)$. The BRCA mutation carriers reported the highest perceived likelihood and the true negatives reported the lowest. Compared to the predisclosure measures, women who received an uninformative DNA test result reported a lower perceived risk after disclosure $(P<0.0001)$, suggesting a relatively high level of reassurance because of the test result. However, after DNA-test disclosure, only 12 women concluded that the risk of carrying a mutation was nonexistent, and perceived likelihood was significantly associated with the pedigree-based risk assessment $(P=0.0001)$. Moreover, despite the significant decrease in perceived likelihood for uninformative women, intention to obtain mammograms did not change $(P=0.71)$; it remained at the same almost optimal level as for BRCA mutation carriers. Conclusion: No support was found for the suggestion that the nature of uninformative test results is often misunderstood. Moreover, an uninformative test result did not affect the positive mammography intentions of both affected and unaffected women. Genet Med 2005:7(4):239-245.
\end{abstract}

Key Words: BRCA1/2 testing, mammography screening, risk perception, uninformative result

Since the isolation of the BRCA1 and BRCA2 genes, many individuals have requested DNA testing for hereditary breast and ovarian cancer. Although reducing uncertainty is mentioned as the prime motive for applying for genetic counseling and DNA testing, ${ }^{1}$ the large majority of test applicants receive a so-called uninformative test result. This is a negative result in the absence of a mutation detected previously within the family. These individuals remain at risk for developing breast cancer based on the pedigree-based risk assessment. Although only a few studies have presented data on this group of test applicants, concern has been expressed about whether women

\footnotetext{
From the ${ }^{1}$ Department of Medical Decision-making, Leiden University Medical Center, Leiden; ${ }^{2}$ Center for Human and Clinical Genetics, Department of Clinical Genetics, Leiden University Medical Center, Leiden; ${ }^{3}$ Department of Social Medicine, Free University, Amsterdam; and the ${ }^{4}$ Department of Clinical Genetics, Erasmus University Medical Center, Rotterdam, The Netherlands.

S. van Dijk, Department of Medical Decision-making, Leiden University Medical Center, PO Box 9600, 2300 RC Leiden, The Netherlands.

Received: May 7, 2004.

Accepted: July 21, 2004
}

DOI: 10.1097/01.GIM.0000159902.34833.26 understand the nature of an uninformative result. It is suggested that women may incorrectly interpret such a result as a true negative result, with possible negative consequences for their surveillance motivation. ${ }^{2-5}$

In concordance with this "false reassurance hypothesis", Hallowell et al. ${ }^{3}$ presented qualitative data describing some affected women who misunderstood the nature of their uninformative result. In addition, Bish et al. ${ }^{2}$ reported a significant decrease in the perceived likelihood of carrying a mutation for affected women who received an interim uninformative DNA test report, and they presume that this may be a sign of incorrect understanding. Although perceived likelihood decreased, no effects on self-reported screening behaviors could be detected. However, with regard to the latter finding, women who are affected with cancer are, generally, under medical supervision already. Indeed, in a population-based study, women who had previously had breast cancer were twice as likely to have had a mammogram compared to unaffected women. ${ }^{6}$ No data are available for screening intentions with regard to unaffected women who have an uninformative test result. The aim of the current study is to assess in more detail whether, and to what extent, an uninformative DNA test result is correctly under- 
stood, and how correct or incorrect interpretations influence surveillance intentions. In a prospective design (i.e., both before and after DNA test disclosure), we compared both affected and unaffected women who received an uninformative test result with: (1) counselees in whom a deleterious BRCA1/2 mutation was detected (i.e., a pathogenic BRCA1 or BRCA2 mutation, which is associated with a lifetime risk of $24 \%$ to $84 \%$ for breast cancer and $11 \%$ to $54 \%$ for ovarian cancer for unaffected women, ${ }^{7-10}$ and (2) participants who tested negative for a $B R C A 1 / 2$ mutation that runs in the family, and who can be considered true negatives, i.e., their lifetime risk reverts to that of the general population.

\section{MATERIALS AND METHODS}

\section{Participants and procedures}

Ethical approval was obtained for the integrated study from both hospitals' research ethics committees. The study comprised all women who were referred for familial breast cancer counseling in the period 1998-2002, met the inclusion criteria of being at least 18 years old, had a sufficient understanding of the Dutch language, and had not having received genetic counseling elsewhere. Referrals for genetic counseling regarding breast cancer were based on current national guidelines. ${ }^{11,12}$ These guidelines specify how many first-degree or second-degree family members should have developed breast cancer either before or after the age of 50, as a prerequisite of referral to a cancer clinic. Genetic counseling was provided by either a clinical geneticist or a genetic nurse. In the first consultation, $B R C A 1 / 2$ testing was offered to individuals if a $B R C A$ mutation had been detected within the family previously, and to individuals where the probability of mutation detection was about $10 \%$ or more, usually an affected family member. The possible results and consequences of $B R C A 1 / 2$ testing were discussed extensively with women who were eligible for genetic testing. After counseling, those women could freely decide whether they would proceed with DNA testing or whether they would ask a family member to take the test for them.

When the DNA test results became available for counselees who actually had decided to have DNA testing, the women concerned were invited to attend a disclosure counseling session of either their own BRCA1/2 test result, or the result of their affected family member. In the latter case, the woman concerned was present, or had provided explicit permission for her result to be discussed with the family member concerned in addition to a personal DNA test disclosure session. As testing of relatives of an uninformative proband is ineffective, unaffected relatives usually had no access to an additional test for themselves. Hence, an uninformative DNA test result of an affected proband was the definitive result for unaffected counselees. Normally, a final familial lifetime risk of developing breast cancer was estimated for women from families in which no $B R C A 1 / 2$ mutation was previously detected..$^{13}$ Four risk categories were distinguished: (1) general population risk, i.e., around $10 \%$; (2) $10 \%-15 \%$; (3) $15 \%-30 \%$; and (4) $30 \%$ or more. Women with an uninformative result were told that they remained at about the same estimated familial lifetime risk, although generally, the likelihood of a high-risk mutation was actually lower after their negative DNA test result. Intensive breast and ovary screening was available for women who proved to have the deleterious $B R C A 1 / 2$ mutation. For all women who received an uninformative test result and had a sufficient strong family history (lifetime risk $\geq 20 \%$ ), intensive breast surveillance was recommended, i.e., annual mammography screening, breast examination by a physician, and monthly breast self-examination. In accordance with current policies for surveillance, ${ }^{14}$ ovary screening was also offered, if cases of ovarian cancer were present in the family history. Women who tested negative for a $B R C A 1 / 2$ mutation that had been detected previously within the family, and women with an uninformative result and a relatively weak family history were informed that intensive surveillance was not recommended. However, they were encouraged to take part in the national population-based screening program for those aged 50 and over. All counselees (including the affected women who provided a blood sample for their family member) were provided with a letter, which summarized all the established information.

All new referrals for breast cancer counseling in Leiden from November 1998, and in Rotterdam from January 2000 until June 2002 were invited to participate in the study by letter. Eligible women who provided written informed consent received questionnaires at various stages. In this study, we report data from the questionnaire immediately after the initial counseling session in which $B R C A 1 / 2$ testing was offered and from the questionnaire that was sent out one month after the provision of the summary letter.

\section{Measures}

Sociodemographic and medical characteristics

All available information about personal history of breast cancer (i.e., unaffected or affected), lifetime risk, $B R C A 1 / 2$ test result, access to intensive breast surveillance, age, educational level, marital status, and number of children was collected.

\section{Perceived likelihood of having inherited a deleterious mutation}

Perception of likelihood as assessed in both questionnaires with the item, "Sometimes you may have asked yourself if you have inherited a characteristic or gene which increases your chances of developing cancer. What do you think the likelihood is that you have inherited such a characteristic/gene? I think that the chance that I have inherited a gene that increases my risk of getting cancer is: 1 'very low' through 4 'neither high nor low' to 7 'very high'." In the second questionnaire, which is after DNA-test disclosure, we expanded the scale to a ninepoint scale with, on both extremes of the scale, the phrases "nonexistent," and "it is certain, the characterictic/gene is detected." This was done to make it possible for women who had now learned that they either carried or did not carry a BRCA1/2 mutation to provide a correct answer. We assumed that the mean perceived likelihood of women with an uninformative 
result would decrease, which would be a correct response. However, for these women the answers "nonexistent" and "certain, the genetic is detected" are incorrect by definition. Hence, we used this item to assess the amount of false reassurance in more detail, and we expected women who would incorrectly interpret their result as a true negative result would provide the answer "nonexistent." In this report, we used perceived likelihood of carrying a deleterious mutation rather than perceived risk of developing breast cancer. Both measures are related, but perceived likelihood of carriership is a more direct measure of comprehension about the nature of the test result, whereas perceived breast cancer risk might resemble a somewhat more global interpretation of several aspects of risk communication and personal risk beliefs.

\section{Intention regarding mammography screening and prophylactic mastectomy}

In both questionnaires, the intention to obtain mammograms was measured with the item "Do you think you will have a mammogram (at least) once every year?" and intention for prophylactic mastectomy was measured with the item "Do you expect to decide to have preventive surgery of your breast(s)." Answers on both items could range from 1 "no, certainly not" through 4 "maybe, maybe not," to 7 "yes, certainly."

\section{Statistical methods}

The SPSS 11.5 statistical package was used to analyze the data. Frequencies were used to describe the study population. We conducted chi-squares and $t$ tests to compare (1) participants who did complete both questionnaires with participants who did not complete both questionnaires, and (2) women with different BRCA1/2 test results, on medical and sociodemographic variables. MANOVAs with repeated measures were used to assess differences between groups and between the pretest and posttest measures for perceived likelihood of carrying a mutation and intention to obtain a mammogram in the forthcoming year. If the groups by DNA test disclosure interaction was significant, simple main effect analyses were conducted. First, the differences between groups were examined separately for the pretest and posttest measures. Secondly, the differences between the pretest and posttest measures were examined in separate groups. The latter analyses were also conducted for the subgroup of unaffected women, as affected women are generally under medical supervision already.

\section{RESULTS}

\section{Study population}

Of the 997 women who met the inclusion criteria, 768 (response rate 77\%: Leiden; $N=657$, Rotterdam; $N=111$ ) consented to participate in the study. Not all women were eligible for DNA testing or chose to have a test. In total, 374 participants received the result of a DNA test as part of their evaluation and counseling. Of those women, 75 did not complete the predisclosure or postdisclosure questionnaire. Of the remaining 299 participants who received a $B R C A 1 / 2$ test result, 12 women were told that a variant of uncertain clinical significance was detected; we will report on these women elsewhere. In addition, 13 women had no remaining breast tissue due to previous surgery (breast cancer surgery and/or prophylactic surgery), and one woman underwent a prophylactic oophorectomy in the period between our measurements. As these surgical procedures are assumed to affect not only the objective cancer risks, but also cancer screening recommendations, we excluded these women from the analyses. This left 273 women for our analyses.

With $t$ tests and chi-square tests, we assessed potential differences between women who completed all measures and women who did not complete either one or both of the questionnaires. No differences were observed for sociodemographic and medical variables (i.e., lifetime risk, $B R C A 1 / 2$ test result, personal history of breast cancer, marital status, educational level, age, having children).

\section{BRCA1/2 test result}

Of the 273 women who received a BRCA1/2 test result, 41 women tested positive for a BRCA1 mutation $(N=32)$ or a $B R C A 2$ mutation $(N=9)$. Of those mutation carriers, 25 carried the deleterious $B R C A 1 / 2$ mutation that had been detected previously within the family, whereas a new BRCA1/2 mutation was found in the other 16 women. In addition, 49 women tested negative for the BRCA1/2 mutation that had been detected previously within the family (BRCA1: $N=34 ; B R C A 2$ : $N=15)$. Finally, 183 women received an uninformative test result; either no mutation was detected in their own blood sample $(N=108)$, or no mutation was found in an affected family member who provided a blood sample on their behalf $(N=75)$.

\section{Sociodemographic and medical characteristics}

Table 1 summarizes the sociodemographic and medical variables of the study population. The mean age of the group was 42.3 years (range 21-72 years; SD 10.6 years), and most women were married or cohabiting and had one or more children. With $t$ tests and chi-squares, we did not observe any differences between the group of test applicants with regard to age, marital status, having children, and level of education. Not surprisingly, percentages of women with a personal history of breast or ovarian cancer were unequally distributed throughout the groups of DNA test results; almost all women with a true negative result were unaffected, whereas in the groups of both BRCA mutation carriers and uninformatives, about half of the women had a prior diagnosis of breast or ovarian cancer. Half of the women who received an uninformative test result remained at a high-risk level ( $\geq 30 \%)$. Of the 89 unaffected women who received an uninformative result, eight women did not have a high enough risk to be eligible for annual mammograms ( $\geq 20 \%$ ), and 11 women were still too young for mammography screening. In addition, four women participated in the national population-screening program (i.e., a mammogram once every 2 years): additional screening was not considered necessary. In total, 66 unaffected women who re- 
Table 1

Sociodemographic and medical variables of the study population $(N=273)$

\begin{tabular}{|c|c|c|c|}
\hline Variable & $\begin{array}{c}B R C A \text { mutation carrier } \\
N=41 \\
\text { No. of women }(\%)\end{array}$ & $\begin{array}{c}\text { True negative result } \\
N=49 \\
\text { No. of women (\%) }\end{array}$ & $\begin{array}{c}\text { Uninformative result } \\
N=183 \\
\text { No. of women (\%) }\end{array}$ \\
\hline \multicolumn{4}{|l|}{ Sociodemographic } \\
\hline \multicolumn{4}{|l|}{ Age } \\
\hline$<30 y$ & $4(10)$ & $8(16)$ & $19(10)$ \\
\hline $30-39$ y & $17(42)$ & $13(27)$ & $50(27)$ \\
\hline $40-49$ y & $8(20)$ & $11(22)$ & $76(42)$ \\
\hline $50+y$ & $12(29)$ & $17(35)$ & $38(21)$ \\
\hline \multicolumn{4}{|l|}{ Marital status } \\
\hline Married or living together & $36(88)$ & $37(75)$ & $154(84)$ \\
\hline Not married or living together & $5(12)$ & $12(25)$ & $29(16)$ \\
\hline \multicolumn{4}{|l|}{ Children } \\
\hline Yes & $28(68)$ & $40(82)$ & $136(74)$ \\
\hline No & $13(32)$ & $9(18)$ & $47(26)$ \\
\hline \multicolumn{4}{|l|}{ Educational level $^{a}$} \\
\hline High school or university & $8(20)$ & $12(25)$ & $56(31)$ \\
\hline Less than high school & $32(80)$ & $36(75)$ & $122(69)$ \\
\hline \multicolumn{4}{|l|}{ Medical } \\
\hline \multicolumn{4}{|l|}{ Breast cancer } \\
\hline Yes & $22(54)$ & $2(4)$ & $94(51)$ \\
\hline No & $19(46)$ & $47(96)$ & $89(49)$ \\
\hline \multicolumn{4}{|l|}{ Objective risk $^{a}$} \\
\hline$<20 \%$ & $\cdots$ & $49(100)$ & $22(13)$ \\
\hline $20-30 \%$ & $\cdots$ & $\cdots$ & $65(40)$ \\
\hline$>30 \%$ & $41(100)$ & $\cdots$ & $78(47)$ \\
\hline \multicolumn{4}{|l|}{ BRCA detected in family } \\
\hline Yes & $16(39)$ & $49(100)$ & $\cdots$ \\
\hline No & $25(61)$ & $\ldots$ & $183(100)$ \\
\hline
\end{tabular}

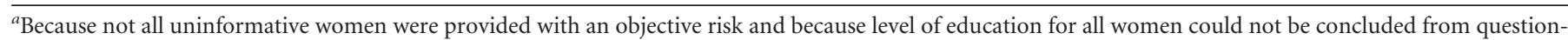
naires, categories do not add up to 273.

ceived an uninformative result were currently eligible for annual mammograms.

\section{Perceived likelihood of carrying a deleterious mutation}

The impact of DNA test disclosure on the perceived likelihood of carrying a mutation was significantly different between groups of test applicants (interaction effect: $\mathrm{F}=193.95$, $P<0.0001)$. Before DNA test disclosure, the three groups differed slightly on the perceived likelihood of carrying a mutation $(\mathrm{F}=3.97, P=0.02)$, with the uninformatives reporting the lowest perceived likelihood (see Table 2). However, after DNA test disclosure, all groups differed greatly from each other $(\mathrm{F}=219.88, P<0.0001)$. All women who learned that they carried the BRCA1/2 mutation shifted to the upper extreme, whereas women who received a true negative result generally shifted to the lower extremes of the scale (see Table 2 for the effects of DNA-test disclosure within groups). Comparable to the true negatives, the women with an uninformative test result perceived the likelihood of carrying a deleterious mutation as being significantly lower after DNA test disclosure.

After disclosure, the group of uninformative women showed a wide variation of responses (Fig. 1), which was significantly correlated with the objective risk estimation, based on the pedigree $(r=0.35, P=0.0001)$. We expected women who would incorrectly conclude that their result was a true negative result, to rate the likelihood of carrying a deleterious mutation as being "nonexistent." Only 10 affected and two unaffected women actually did choose this response (6.6\%). Six of the women who chose this response had no elevated risk or a relatively low risk, whereas the other six women had either 
Table 2

Perceived likelihood and screening intention before and after DNA test disclosure

\begin{tabular}{|c|c|c|c|c|c|}
\hline & \multicolumn{3}{|c|}{ Mean (SD) } & \multicolumn{2}{|c|}{ Effect disclosure within groups } \\
\hline & $N$ & Predisclosure & Postdisclosure & $\mathrm{F}$ & $P$ \\
\hline \multicolumn{6}{|l|}{ Perceived risk carriership $(0-8)$} \\
\hline BRCA mutation carrier & 41 & $5.37(1.43)^{b}$ & $8.00(0.00)^{c}$ & 97.96 & $<0.0001$ \\
\hline True negative result & 49 & $5.14(1.14)^{a b}$ & $0.67(1.48)^{a}$ & 337.03 & $<0.0001$ \\
\hline Uninformative result & 181 & $4.79(1.34)^{a}$ & $3.50(1.88)^{b}$ & 102.84 & $<0.0001$ \\
\hline \multicolumn{6}{|l|}{ Intention mammography (1-7) } \\
\hline BRCA mutation carrier & 40 & $6.18(1.55)^{b}$ & $6.20(1.80)^{b}$ & 0.01 & 0.922 \\
\hline True negative result & 46 & $5.54(1.62)^{a}$ & $2.74(1.90)^{a}$ & 139.93 & $<0.0001$ \\
\hline Uninformative result & 173 & $6.25(1.38)^{b}$ & $6.30(1.37)^{b}$ & 0.14 & 0.706 \\
\hline Uninformative subgroup $^{e}$ & 66 & $6.29(1.33)$ & $6.55(0.86)$ & 1.52 & 0.220 \\
\hline
\end{tabular}

$\overline{a b c}$ If in the column predisclosure or postdisclosure group means do not share a similar superscript they differ significantly at $P<0.05$ level.

${ }^{e}$ Intention for the subgroup of uninformative women who were unaffected and eligible for mammography.

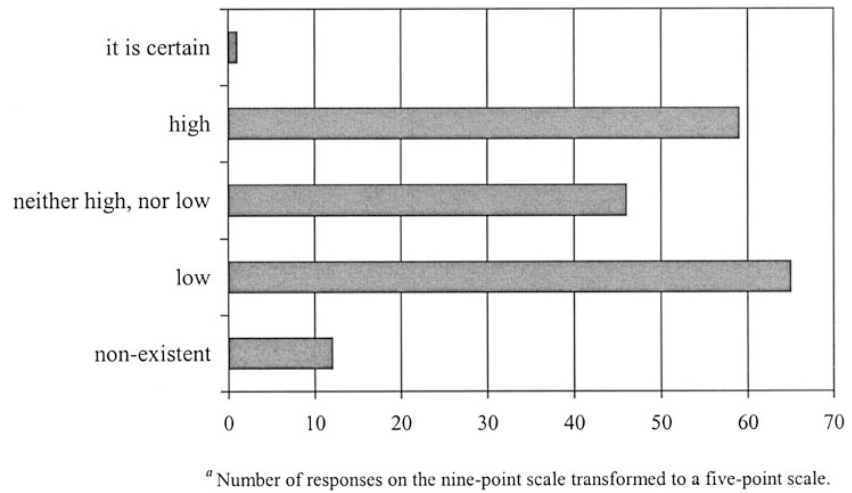

Fig. 1. Perceived likelihood of carrying a deleterious mutation after receiving an uninformative BRCA1/2 test result.

a moderately increased risk or a highly increased risk. Finally, one woman incorrectly stated that the deleterious mutation was detected. She had only a moderately increased risk (i.e., $15 \%-30 \%)$. However, she suffered from mastopathy and already reported a strong desire for prophylactic mastectomy in the first consultation, which might be a motivational reason to interpret her uninformative result as a proof of carriership.

\section{Intention regarding mammography screening}

The impact of DNA-test disclosure on the intention to obtain mammograms within the next year was significantly different between groups of test applicants (interaction effect: $\mathrm{F}=$ 59.29, $P<0.0001)$. Before disclosure, the three groups already had a significantly different screening intention $(\mathrm{F}=4.33, P=$ 0.014 ), with women with a true negative result having a significantly lower intention than BRCA1/2 mutation-carriers and uninformatives (Table 2). However, compared to the other groups, the group of true negatives contained very few women who were under medical supervision, due to a previous diagnosis of breast cancer. Therefore, we examined whether the observed predisclosure difference was due to this variation.
Indeed, if we selected only unaffected respondents for the same analysis, the groups did not differ on intention regarding mammography screening before DNA test disclosure ( $\mathrm{F}=$ $1.87, P=0.16$ ).

After disclosure, the three groups differed very significantly with regard to screening intention $(\mathrm{F}=99.22, P<0.0001)$, and this effect remained very strong if we selected only unaffected women $(\mathrm{F}=68.31, P<0.0001)$. BRCA mutation carriers and uninformatives did not change their intention after disclosure, whereas women with a true negative result reported a highly significant decrease in intention, in accordance with their now negative indication for annual mammography (see Table 2).

With regard to the uninformatives, 151 out of 173 women (87\%) reported a positive intention. If we selected the unaffected women who were currently eligible for annual mammography $(N=66)$, the results were very compatible with the overall results; the overall level of mammography screening intention remained at a high level, with even 63 out of 66 women (95\%) reporting a positive intention after DNA test disclosure (Table 2). For the 12 uninformative women who seemed to interpret their result incorrectly as a true negative result (i.e., "the likelihood is nonexistent"), we inspected responses regarding the intention to have at least annual mammograms; 11 women had a positive intention (i.e., score $\geq 5$ ), whereas one woman was undecided $($ score $=4)$. Thus, the potential false reassurance did not clearly result in a failure to adhere to regular screening.

The intention to have a yearly mammogram remained very strong among $B R C A$ mutation carriers ( $88 \%$ reported a positive intention). Four carriers reported a (somewhat) negative intention (i.e., score $\leq 3$ ), and one woman was undecided after DNA test disclosure (score $=4$ ). To check whether the choice for prophylactic mastectomy as an alternative risk management option would explain negative intentions, we excluded 19 women who had decided to undergo prophylactic mastec- 
tomy (i.e., they said that they would definitely have prophylactic mastectomy). After this selection, all BRCA mutation carriers were found to have an optimal positive intention to obtain annual mammograms $(M=6.90 ; \mathrm{SD}=0.30)$.

In general, women with a true negative result reported a negative intention after BRCA1/2 testing, which seemed appropriate given the subsequent contraindication for intensive surveillance. However, 8 out of 46 women (17\%) mentioned a (somewhat) positive intention. For six of them, this seemed to be quite understandable, as they were under medical supervision due to a previous breast cancer $(N=1)$ or mastopathy $(N$ $=1$ ), or were taking part in the national population-screening program $(N=4)$. For two women who received a true negative result, it remained unclear from a medical point of view why they would opt for intensive breast surveillance.

\section{DISCUSSION}

In several studies, concern has been expressed about the possible ambiguity of an uninformative DNA test result for breast cancer. Women might incorrectly interpret this as a true negative result, with possible negative consequences for their adherence to surveillance recommendations. In the current prospective clinic-based sample of $B R C A 1 / 2$ test applicants, we found a strong indication that either a familial or personal uninformative test result might provide reassurance with regard to their perceived likelihood of carriership. However, we did not find evidence that this reassurance was due to a lack of understanding of the nature of an uninformative DNA test result. Perceived likelihood of carrying a deleterious mutation decreased significantly after DNA-test disclosure for women with an uninformative result, which can be considered appropriate, as the likelihood of a high penetrance mutation is actually smaller after an uninformative result. However, the perceived likelihood of carriership was not only significantly different from that of $B R C A$ mutation carriers, but also from that of true negatives. Moreover, only a very small minority concluded that the likelihood of a deleterious mutation is nonexistent after DNA test disclosure.

For the whole group, the pretest level of intention for obtaining mammograms was rather high. After test DNA test disclosure, true negatives significantly decreased their intention, whereas the intentions of $B R C A$ mutation carriers and the overall group of uninformative women remained stable. The lack of change in the latter two groups is likely attributable to the high baseline mammography screening intention. We also found that unaffected uninformatives did not change their strong intention. This is important, as the single study that reported on (a high rate of) mammography utilization among women who receive uninformative test results was restricted to affected women. ${ }^{2}$ Unlike affected women, unaffected women, who learn that they are eligible for screening due to their increased cancer risk, are generally not included in a standard surveillance protocol yet. Thus, it is reassuring that their mammography intentions remain very positive after DNA test disclosure.
Regarding screening behaviors, recently concern has been expressed about a possible suboptimal utilization of surveillance options for BRCA1/2 mutation carriers. ${ }^{15,16}$ Reports of mammography uptake among proven mutation carriers vary from $59 \%{ }^{15}$ to $88 \% .{ }^{17}$ In the current study, we observed a very strong intention toward having a mammogram for both the groups of uninformative women and BRCA1/2 mutation carriers. Moreover, if we controlled for a very positive intention for prophylactic mastectomy among the BRCA1/2 mutation carriers, all carriers expressed a very positive intention. Thus, in this report about intention, rather than actual behavior, we do not find reasons for concern.

In a recent study, adherence to mammography proved to be strongly associated with physicians' recommendations. ${ }^{17} \mathrm{We}$ hypothesize that this might be an explanation for our very positive screening intentions, as in our clinics in both Leiden and Rotterdam, multidisciplinary medical care is available, especially for women with a strong family history of cancer. In both clinics, high-risk women are encouraged to opt for intensive screening and in Leiden, a first appointment for a mammogram was even automatically scheduled after the surveillance recommendations of the clinical geneticist. Thus, our results support the suggestion of Tinley et al., ${ }^{17}$ that education and support for screening from primary providers might be (part of) a clinical solution for optimization of adherence to screening. Another explanation for the positive screening intentions might be that mammography screening is relatively easy to obtain within the Dutch health care system. Different intentions might be observed in a system where access to screening services is dependent upon availability, or ability to pay.

A few limitations of the current study must be noted. First of all, although intention to have a mammography is a main predictor for actual utilization, ${ }^{18}$ intentions for mammography, even very strong intentions, as in our sample, might not always translate into actual behavior. Follow-up data are needed to check whether actual utilization remains as strong among the group of $B R C A 1 / 2$ mutation carriers and women who receive an uninformative result. Secondly, the few women who report that the likelihood of carrying a mutation is "nonexistent" or "certain," in the face of an uninformative result, do not necessarily misunderstand such a result. These women may simply not believe or accept the message. In this respect, it is interesting that the woman with an uninformative result, who stated incorrectly that the deleterious mutation was found, was probably psychologically motivated to interpret her result this way; she desired a prophylactic mastectomy, because of her anxiety about developing breast cancer. Furthermore, uninformative women who do not rate the likelihood as "nonexistent" or "certain," do not necessarily understand their result in a proper way.

However, given the perceived likelihood and the mammography intentions reported, we do not think there is a tendency to interpret an uninformative result as a true negative result. Apparently, genetic counseling is effective in assisting women in understanding their DNA test result. Moreover, an uninfor- 
mative test result also had no negative impact on screening intention.

\section{ACKNOWLEDGMENTS}

The Dutch Cancer Society (UL 98-1740) financially supported this study. Thanks are due to all the women who participated.

\section{References}

1. Van Asperen CJ, Van Dijk S, Zoeteweij MW, Timmermans DR, De Bock GH, Meijers-Heijboer EJ, Niermeijer MF et al. What do women really want to know? Motives for attending familial breast cancer clinics. J Med Genet 2002;39:410-414.

2. Bish A, Sutton S, Jacobs C, Levene S, Ramirez A, Hodgson S. No news is (not necessarily) good news: impact of preliminary results for $B R C A 1$ mutation searches. Genet Med 2002;4:353-358.

3. Hallowell N, Foster C, Ardern-Jones A, Eeles R, Murday V, Watson M. Genetic testing for women previously diagnosed with breast/ovarian cancer: Examining the impact of BRCA1 and BRCA2 mutation searching. Genet Test 2002;6:79-87.

4. Couch FJ, Hartmann LC. BRCA1 testing: Advances and retreats. JAMA 1998 25;279: 955-957.

5. Lerman C, Marshall J, Audrain J, Gomez-Caminero A. Genetic testing for colon cancer susceptibility: Anticipated reactions of patients and challenges to providers. Int J Cancer 1996;69:58-61.

6. Legg JS, Fauber TL, Ozcan YA. The influence of previous breast cancer upon mammography utilization. Womens Health Issues 2003;13:62-67.

7. Antoniou A, Pharoah PD, Narod S, Risch HA, Eyfjord JE, Hopper JL, Loman N et al. Average risks of breast and ovarian cancer associated with BRCA1 or BRCA2 mutations detected in case Series unselected for family history: A combined analysis of 22 studies. Am J Hum Genet 2003;72:1117-1130.
8. King MC, Marks JH, Mandell JB; New York Breast Cancer Study Group. Breast and ovarian cancer risks due to inherited mutations in BRCA1 and BRCA2. Science 2003;302:643-646.

9. Ford D, Easton DF, Stratton M, Narod S, Goldgar D, Devilee P et al. Genetic heterogeneity and penetrance analysis of the BRCA1 and BRCA2 genes in breast cancer families. The Breast Cancer Linkage Consortium. Am J Hum Genet 1998;62:676689.

10. Easton DF, Ford D, Bishop DT. Breast and ovarian cancer incidence in BRCA1mutation carriers. Breast Cancer Linkage Consortium. Am J Hum Genet 1995;56: 265-271.

11. De Bock GH, Vliet Vlieland TP, Hageman GC, Oosterwijk JC, Springer MP, Kievit J. The assessment of genetic risk of breast cancer: A set of GP guidelines. Fam Pract 1999;16:71-77.

12. Van Asperen CJ, de Bock GH, van der Horst F, de Koning HJ, Rutgers EJ. Screening for breast cancer on basis of individual risk assessment for women ineligible for the national population screening program. Ned Tijdschr Geneeskd 2001;145:120-125.

13. Claus EB, Risch N, Thompson WD. Autosomal dominant inheritance of early onset breast cancer. Cancer 1994;73:643-651.

14. Vasen HF, Haites NE, Evans DG, Steel CM, Moller P, Hodgson S, Eccles D et al. Current policies for surveillance and management in women at risk of breast and ovarian cancer: a survey among 16 European family cancer clinics. European Familial Breast Cancer Collaborative Group. Eur J Cancer 1998;34:1922-1926.

15. Peshkin BN, Schwartz MD, Isaacs C, Hughes C, Main D, Lerman C. Utilization of breast cancer screening in a clinically based sample of women after BRCA1/2 testing. Cancer Epidemiol Biomarkers Prev 2002;11:1115-1118.

16. Botkin JR, Smith KR, Croyle RT, Baty BJ, Wylie JE, Dutson D et al. Genetic testing for a BRCA1 mutation: Prophylactic surgery and screening behavior in women 2 years post testing. Am J Med Genet 2003;118A:201-209.

17. Tinley ST, Houfek J, Watson P, Wenzel L, Clark MB, Coughlin S et al. Screening adherence in BRCA1/2 families is associated with primary physicians' behavior. Am J Med Genet 2004;125A:5-11.

18. Hofvind SS, Wang H, Thoresen S. The Norwegian Breast Cancer Screening Program: re-attendance related to the women's experiences, intentions and previous screening result. Cancer Causes Control 2003;14:391-398. 